\title{
Consumo televisivo y nivel de interacción y participación social en redes sociales: análisis de las audiencias millennials en la campaña electoral de España
}

\author{
Television consumption and level of social interaction \\ and participation on social networks: an analysis of \\ millennial audiences in the Spanish electoral campaign
}

\author{
Cristina González-Oñate; Gloria Jiménez-Marín; Paloma Sanz-Marcos
}

Cómo citar este artículo:

González-Oñate, Cristina; Jiménez-Marín, Gloria; Sanz-Marcos, Paloma (2020). “Consumo televisivo y nivel de interacción y participación social en redes sociales: análisis de las audiencias millennials en la campaña electoral de España". Profesional de la información, v. 29, n. 5, e290501.

https://doi.org/10.3145/epi.2020.sep.01

Artículo recibido el 10-03-2020 Aceptación definitiva: 04-05-2020

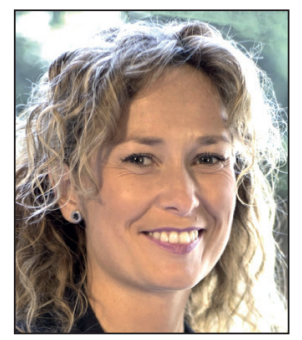

\author{
Cristina González-Oñate $\triangle$ \\ https://orcid.org/0000-0003-3509-0117 \\ Universitat Jaume I \\ Depto. de Ciencias de la Comunicación \\ Av. de Vicent Sos Baynat, s/n. \\ 12071 Castellón, España \\ onate@uji.es
}

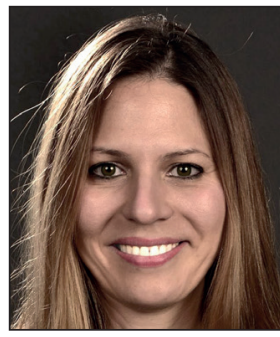

Gloria Jiménez-Marín

https://orcid.org/0000-0003-0252-3975

Universidad de Sevilla

Depto. de Comunicación audiovisual y

Publicidad

Av. Américo Vespucio, s/n.

41092 Sevilla, España

gloria_jimenez@us.es

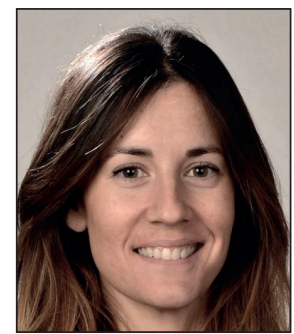

\author{
Paloma Sanz-Marcos \\ https://orcid.org/0000-0002-6103-6993 \\ Universidad de Sevilla \\ Depto. de Comunicación audiovisual y \\ Publicidad \\ Av. Américo Vespucio, s/n. \\ 41092 Sevilla, España \\ palomasanz@us.es
}

\section{Resumen}

En un contexto en el que el espectador es considerado adicto a sus dispositivos móviles y multipantallas, la combinación entre mensajería instantánea, televisión y redes sociales resulta interesante para analizar la interacción y la compartición de contenidos. Ese panorama también viene impulsado por la propia televisión, que ha empezado a introducir de manera estratégica la participación dentro de los propios contenidos con el fin de dar protagonismo al espectador. La televisión vive un momento de esplendor y lleno de oportunidades con nuevos negocios. Bajo esta premisa, resulta de gran interés analizar y estudiar el nivel de interacción y de participación que han tenido las audiencias millennials en las pasadas elecciones políticas, no sólo en redes sociales, sino también por medio de otras pantallas, especialmente la televisiva. Se analiza el comportamiento de jóvenes universitarios españoles respecto a los contenidos políticos televisivos y digitales para conocer sus preferencias y la efectividad de los mismos en relación al voto. La idea principal es conocer cómo ha sido el nivel de participación e interacción que han tenido respecto a los mensajes políticos en redes y en televisión, y si su comportamiento ha tenido o no un efecto en sus decisiones dentro de las urnas. Para ello se ha utilizado la encuesta como herramienta principal en varias comunidades autónomas con el objetivo de comprobar si hay diferencias entre ellas para el objeto de estudio. 


\title{
Palabras clave
}

Audiencias; Consumo; Contenidos; Elecciones; Estrategia; Interacción; Millennials; Participación; Política; Comunicación política; Redes sociales; Medios sociales; Televisión.

\begin{abstract}
In a context in which the audience is considered to be addicted to their mobile and multi-screen devices, the combination between instant messaging, television, and social networks is very interesting regarding the interaction and social participation with content. This effect is also driven by television itself, which has begun to strategically introduce participation within the content itself to give prominence to the audience. Television is enjoying a moment of splendor that is full of opportunities for new businesses. It is of great interest to analyze and study the level of interaction and participation of millennial audiences with past political elections, through the study of not only social networks but also other screens, especially television. In this research, the behavior of young Spanish university students regarding television and digital political content is analyzed to determine their preferences and the effectiveness of the content regarding their vote. The main aim is to determine the level of social participation and interaction of these audiences with political messages on networks and television, and whether such behavior affects their voting decisions. To this end, a survey was carried out in various Spanish autonomous communities to confirm whether differences were present.
\end{abstract}

\section{Keywords}

Audiences; Consumption; Contents; Elections; Interaction; Millennials; Participation; Politics; Strategy; Political communication; Social networks; Social media; Television.

\section{Introducción}

Nos encontramos en un nuevo escenario televisivo caracterizado por un aumento muy significativo de la oferta de contenidos, por un uso cada vez mayor de otros dispositivos multipantalla y, especialmente, por el surgimiento de nuevos hábitos a la hora de acceder y de consumir estos contenidos (Castells, 2007). Estamos asistiendo a un cambio en la manera en que la sociedad se relaciona y accede a los contenidos (Dalton, 2004), donde la interacción y la participación cobran protagonismo.

En este contexto mediático, las audiencias se han dotado de mayores posibilidades de interacción con los contenidos televisivos gracias a los avances tecnológicos y a la cultura de la participación (Jenkins; Ito; Boyd, 2015). Las redes sociales y las nuevas plataformas de emisión han posibilitado nuevos canales de comunicación que permiten incorporar la interactividad de los usuarios, así como la existencia de un feedback entre emisor y receptor (González-Neira; Quintas-Froufe, 2015). La actitud pasiva y lineal del espectador se convierte en activa y experiencial en la denominada televisión interactiva o social (González-Neira; Berrocal-Gonzalo; Zamora-Martínez, 2020), caracterizada por ser bidireccional, personalizada y por conseguir mitigar necesidades de entretenimiento y comunicación (León; García-Avilés, 2012).

La interacción de la audiencia equivale en síntesis a (Perilla-Lozano; Zapata-Cadavid, 2009):

- visibilizar los vínculos y su utilidad entre las personas, los grupos y las organizaciones;

- estimular metodologías que promuevan la participación democrática, sin forzarla ni imponerla;

- identificar los significados que emergen en las relaciones y contribuir a su transformación.

En cambio, la participación de la audiencia se percibe no únicamente como activa en la interpretación de los contenidos, sino también en la producción y el debate de los mismos (Masip; Ruiz-Caballero; Suau, 2019), incluso utilizando las plataformas sociales para comentar e influir en la emisión en directo de la programación televisiva. La participación social, por tanto, es un tipo de interacción que implica relación con otros participantes provocando la posible repetición de la acción (Castromil; Rodríguez-Díaz; Garrigós, 2020).

La televisión social, caracterizada fundamentalmente como canales alternativos como las redes sociales y segundas y terceras pantallas que sirven de soporte conversacional en torno a lo que sucede en una programación (Gallego, 2013), ha provocado la existencia de conversación en tiempo real entre usuarios distanciados físicamente y un intercambio vertical entre el emisor y el receptor (Dias; Teixeira-Botelho, 2016). La tecnología ha generado nuevas narrativas para llegar al consumidor (Quintas-Froufe; González-Neira, 2014), pero también ha abierto nuevas posibilidades, que han aumentado en número de competidores a la hora de captar la atención del usuario. Se han incorporado nuevas cadenas, plataformas digitales televisivas y nuevos géneros a la oferta tradicional de contenidos (Scolari et al., 2012). La mayor oferta se ha correspondido con una mayor adscripción y fidelidad de los espectadores. Y las redes sociales han contribuido a este aumento del consumo porque han facilitado la interacción en tiempo real (Cabrera-González, 2010) y la participación en el contenido por parte del espectador (Guerrero-Pérez; González-Oñate; Kimber-Camussetti, 2018).

La tecnología ha modificado los hábitos de los ciudadanos en todas las facetas de la vida (De-la-Garza-Montemayor; Peña-Ramos; Recuero-López, 2019). Esta nueva realidad propicia que los propios medios digitales se configuren como una alternativa y como un nuevo agente de socialización (Grossman, 1995), especialmente en el ámbito de la comunicación política que, mediante la combinación de las redes sociales con otras pantallas como la televisiva (Francés-Domenec; 
Llorca-Abad; Peris-Blanes, 2015) se dirige hacia un tipo de comunicación mucho más híbrida (Rodríguez-Vázquez et al., 2020). La televisión ya no puede ser ajena a todos los cambios que la tecnología produce en nuestros hábitos de consumo y estilos de vida. Es la magia de la tecnología aplicada a la mejora de la sociedad de consumo (Oliver; Alloza, 2009). Por ello, las empresas televisivas (Bustamante, 2006) se han visto obligadas a modificar la forma de relacionarse con los consumidores, la manera de comunicar sus contenidos y, sobre todo, ver en internet una nueva vía de difusión más adecuada para audiencias de carácter juvenil.

Actualmente las audiencias están muy fragmentadas, los anunciantes empiezan a pagar por contenidos y además el consumo se realiza desde distintos dispositivos y momentos (Benavides-Delgado et al., 2010). Pese a todos estos cambios, la televisión sigue siendo el motor de la notoriedad publicitaria y prueba de ello son los datos de inversión en este medio que sigue siendo el rey. Llevamos muchos años escuchando que la televisión se acababa con la llegada de internet o que iba a perder eficacia en términos de publicidad, pero la televisión también ha evolucionado y su poder de influencia sigue siendo el mismo o incluso mayor (Campos-Freire, 2015)

Internet ha propiciado nuevos usos de la televisión otorgando al espectador la opción de elegir su consumo cómo y dónde quiera (Quintas-Froufe; González-Neira, 2016). Televisión e internet no pueden ser considerados medios excluyentes, sino que se integran (Ferreras-Rodríguez, 2014). Esta multiconectividad ha cambiado el lugar destacado de ocio que ocupaba el televisor cediendo el paso a las segundas y terceras pantallas (Vaca, 2015), animando a la audiencia a interactuar con la oferta y a que participe con los contenidos compartiendo experiencias con otros usuarios y consiguiendo así un feedback en directo (Ferguson; Greer, 2016).

La televisión es una plataforma más potenciada por los medios digitales que generan un efecto amplificador de la audiencia televisiva (Waddell; Bailey, 2017), siendo el consumo de contenidos muy dispar según los dispositivos, por lo que ya no importa tanto el dónde se consuma sino el cómo (Diego-González; Guerrero-Pérez; Etayo-Pérez, 2014). La televisión es un medio que debe seguir avanzando en términos de negocio digital (Gallego, 2013), ya no sólo por una cuestión económica, sino también por temas de eficacia para conseguir cobertura y notoriedad a la hora de publicitar marcas comerciales y con el fin de extraer un mayor rendimiento y explorar nuevas conexiones con el usuario (Saavedra-Llamas; Papi-Gálvez; Perlado-Lamo-de-Espinosa, 2020).

\section{Nuevos hábitos de visionado: Consumidores multipantalla, light TV viewers y millennials televisivos}

La tecnología ha hecho que las personas evolucionen a una velocidad vertiginosa (Campos-Freire, 2010), por lo que surgen nuevas generaciones con identidades, necesidades, hábitos de comportamiento y de consumo muy característicos (Masip et al., 2015). Una velocidad que se hace más latente entre las audiencias más jóvenes (Quintas-Froufe; González-Neira, 2014).

Los avances tecnológicos nos permiten realizar varias tareas a la vez, especialmente cuando estamos en movimiento. En este sentido, el consumidor actual, o bien ha nacido ya con este tipo de multitarea, los denominados nativos digitales (Prensky, 2001), o bien se ha acostumbrado a ella (Li; Bernoff, 2009). Aprovechamos cualquier desplazamiento para buscar información sobre productos, consultar redes sociales, revisar y contestar correos electrónicos, etc. El concepto de tiempo se ha vuelto más importante en nuestras vidas y buscamos en la tecnología la manera de rentabilizarlo.

El espectador medio de la televisión convencional ha pasado a ser creador y consumidor -prosumer- (Gil; Romero, 2008), y ha evolucionado desde un consumidor pasivo a su consideración como usuario activo que interactúa y participa por mediación de internet, tanto en el televisor, como en los dispositivos adyacentes (Cebrián-Herreros, 2009). Hemos pasado de individuos aislados a usuarios híper conectados que buscan experiencias únicas, contenido de interés e inmediatez en todos los procesos (Prensario, 2018).

Este escenario nos ha permitido identificar tres tipos de espectadores con una serie de rasgos característicos que, a pesar de estar muy próximos entre sí, incluyen diferencias significativas:

- los consumidores multipantalla;

- los denominados light TV viewers;

- los millennials televisivos.

El consumidor multipantalla tiene un perfil dinámico y activo, pero a su vez sin concentración (Hershatter; Epstein, 2010). Es decir, la atención del consumidor frente a la pantalla del televisor está cada vez más fragmentada y, al mismo tiempo, es más intermitente, puesto que es compartida con otros dispositivos: programas de televisión comentados en redes sociales, entretenimiento votado en otras plataformas, series de televisión consumidas en varias pantallas, concursos e incluso debates políticos llevados a otros debates en otras pantallas.... Son grandes consumidores digitales y grandes generadores de información (Espinoza; Ukleja; Rusch, 2010). Los contenidos se consumen casi de manera simultánea, la interacción y otras opciones de comunicación se producen a la vez (Cabrera-González, 2010).

El concepto de tiempo se ha vuelto más importante en nuestras vidas y buscamos en la tecnología la manera de rentabilizarlo 
Los usuarios multipantalla ven contenido en la televisión y al mismo tiempo utilizan otros dispositivos, ya sea para generar contenidos sobre el programa que están viendo, o simplemente para interactuar con otros usuarios (DeVaney, 2015). Este hecho se hace evidente cada vez que un programa de televisión emite contenido relacionado, por ejemplo, con redes sociales. Además, hay segmentos de edad que ven menos televisión convencional y se inclinan por un consumo a la carta. Los usuarios pasan a ser espectadores sociales que interactúan de manera activa con el contenido televisivo, comentan los programas con otros espectadores y buscan otros comentarios sobre el programa. Es la denominada televisión social, cuyo escenario multipantalla multiplica las posibilidades de impacto para captar la atención y aumentar la retención de un consumo audiovisual concreto (Castelló-Martínez; Del-Pino-Romero; Tur-Viñes, 2016).

El consumidor multipantalla requiere y exige a las marcas televisivas inmediatez en un medio que evoluciona de manera constante (González-Oñate, 2008). Este perfil de usuario puede realizar múltiples tareas de manera simultánea, y decide los canales por los que quiere interactuar con sus marcas (Marta-Lazo; Gabelas-Barroso, 2013). La calidad informativa toma el relevo (Tur-Viñes, 2020) y se hace indispensable como herramienta de integración de los diferentes canales en tiempo real, generando con ello trazabilidad de las interacciones y la aplicación de procesos de conocimiento del consumidor (Casero-Ripollés, 2018).

Para este tipo de público, el contenido audiovisual tiene que ofrecer la misma experiencia positiva independientemente de en qué plataforma se consuma. Sólo los contenidos que el consumidor haya elegido en el momento, así como su calidad, captarán su atención lo suficiente para que ese consumo se alargue en el tiempo. Por tanto, la televisión necesita planes integrados que incluyan soportes digitales para generar sinergias significativas entre otras pantallas (Waddell; Sundar, 2017). Este aspecto provoca a su vez una pérdida de atención y, en consecuencia, una pérdida de recuerdo.

Según el estudio Connected Life, elaborado por Kantar TNS a finales de 2014 (Kantar TNS, 2014), podemos determinar cuatro personalidades de los consumidores multipantalla:

- Líderes: el número de dispositivos que poseen los líderes puede ser mayor en los mercados más acomodados -como el europeo o las regiones más desarrolladas de Asia- que en muchos de los mercados asiáticos y africanos, por ejemplo. Sin embargo, los líderes siempre destacan por su participación constante y enérgica en el mundo virtual, por su curiosidad por la tecnología y por su elevada dependencia de ella. Y no se trata, en absoluto, de un grupo nicho. Los líderes constituyen habitualmente un tercio de la población online en todos los mercados.

- Funcionales: habitualmente suponen otro tercio de la población digital en la mayoría de los mercados. Adoptan la tecnología con mayor lentitud y precaución, a menudo obligados por la necesidad de mantenerse al día respecto al mundo, o bien porque son conscientes de que proporciona unos beneficios prácticos tangibles.

- Observadores: sienten una gran curiosidad y entusiasmo por la tecnología y se enorgullecen de conocer las últimas innovaciones. Se sienten enormemente cómodos con la tecnología y disfrutan siendo capaces de entender las cosas por sí mismos. Lo que les diferencia de los líderes es su falta de entusiasmo por las redes sociales.

- Conectores: son lo contrario de los observadores, ya que su interacción con el mundo online se debe principalmente a las redes sociales. Tienden a representar el 15-20\% de la población online en la mayoría de los mercados. No están especialmente interesados en tener los últimos dispositivos, siempre que los que tengan sean suficientemente buenos para acceder a las redes sociales y conectarse con la familia y los amigos.

El crecimiento de internet nos lleva a un nuevo ecosistema de medios (Crovi, 2004) y a un nuevo consumidor de televisión: los denominados light TV viewers que tienden a ser jóvenes o parejas jóvenes (con o sin niños) de entre 18 y 34 años, con estudios universitarios, y de clase social media, media-alta, que se caracterizan por ser influyentes y claros prescriptores en las redes sociales (Valiente, 2016). Este perfil ve menos de una hora y media de televisión al día (unos 75 minutos) frente a las cuatro horas de promedio, y comprenden aproximadamente el $20 \%$ de la población. Esta nueva forma de consumo de televisión es especialmente acusada en este público, cuyas frecuencias de impacto con la televisión se ven complementadas en el entorno online. Son consumidores activos, siempre conectados (Bermejo-Berros, 2008), que generan contenidos, demandan interactividad social y viral y buscan engagement en las marcas televisivas (Chen et al., 2015).

El light TV viewer tiene un consumo de televisión menor porque prefiere otros medios de comunicación para estar informado de la actualidad. En cambio, el anterior perfil descrito (el consumidor multipantalla), consume televisión para estar informado y, a su vez, realiza otras actividades en otras pantallas, especialmente, en redes sociales y con su teléfono móvil o tableta (Pérez-Torres; Pastor-Ruiz; Abarrou-Ben-Boubaker, 2017). Los patrones de uso son variados y evolucionan de manera rápida, pero en este perfil la segunda pantalla es ya un fenómeno simultáneo en el momento de consumir televisión e incluso generalizado en diferentes franjas de edad, ya que el teléfono móvil interrumpe con fuerza cuando se consume televisión, ya sea por entretenimiento o por estar informados (Guerrero-Pérez; González-Oñate; Kimber-Camussetti, 2018).

Por último, están los denominados millennials, también conocidos como la "generación Y" que relevan a la "generación X" y los denominados baby boomeers (Beauchamp; Barnes, 2015), jóvenes entre 18 y 28 años que han crecido con las nuevas tecnologías y no recuerdan un mundo sin internet, cuya extensión experimental pasa por los smartphones y las redes sociales principalmente (Álvarez-Monzoncillo; López-Villanueva, 2017). Buscan constantemente nuevas expe- 
riencias comunicativas más personalizadas, contenidos originales y salir de lo común, elementos con los que se identifiquen y que les proporcionen la oportunidad de participar en la conversación. Buscan un estímulo que les impulse a compartir sus ideas y sus experiencias en las redes sociales con sus amigos (Ruiz-Cartagena, 2017), sobre todo, a través de su teléfono móvil, herramienta indispensable para ellos (González-Neira; Quintas-Froufe, 2015).

De entre los rasgos que definen a este público destacan:

- alta exposición a la tecnología y a la información (Prensky, 2001);

- necesidad de socialización y de conexión (Barton; Koslow; Beauchamp, 2014);

- destacado comportamiento multiplataforma y multitarea (Hershatter; Epstein, 2010).

Asimismo, se caracterizan por tener un buen nivel de formación (universitario), son socialmente comprometidos, colaborativos y pragmáticos, pero a su vez también son muy inquietos, muy emocionales y críticos, lo que les lleva a ser exigentes (Ruiz-Cartagena, 2017). Este colectivo es la revolución del mundo digital actual y requiere estrategias específicas por parte de las marcas para captar su atención (Valiente, 2016).

Respecto a su relación con la televisión, hemos de resaltar que los millennials buscan experiencias personalizadas, contenidos originales y, sobre todo, que sean diferentes para después poder compartirlas en las redes sociales (Guerrero-Pérez; González-Oñate; Kimber-Camussetti, 2018). Reivindican formar parte del contenido televisivo, identificarse con el mensaje que las marcas les proporcionen en varios de sus dispositivos, especialmente, en sus smartphones (De-Salas-Nestares, 2010). Los millennials han adoptado servicios bajo demanda como parte integrada en sus rutinas. La posibilidad de elegir el contenido que les interesa en cada momento está llevando a muchas empresas a incorporar soluciones adaptadas a los requerimientos de los consumidores (Valiente, 2016). Esto está transformando a muchas empresas que están diseñando servicios a demanda y convirtiéndolos en valores estratégicos para sus organizaciones (Cebrián-Herreros, 2009).

Finalmente, cabe destacar que los millennials televisivos combinan la interacción y el consumo mediante YouTube como plataforma audiovisual, aspecto especialmente interesante, puesto que hacen uso de esta plataforma sobre todo cuando están frente al televisor. El poder de influencia de YouTube sobre este target ha generado un nuevo perfil publicitario que muchas marcas están empezando a explotar como vehículo y como reclamo de la atención de este tipo de consumidor televisivo (Cunningham; Craig; Silver, 2016).

\section{Objetivos y metodología}

El objetivo principal de este trabajo es analizar el comportamiento de las audiencias millennials en las diferentes pantaIlas. Principalmente, se pretende estudiar cómo han consumido contenidos de carácter político durante las elecciones de 2019 en España, sobre todo en televisión y redes sociales, para comprobar el nivel de eficacia de los mensajes y contenidos políticos para este público, así como el nivel de interacción, participación y conexión entre las diferentes pantallas. Con contenidos políticos nos referimos principalmente a debates electorales, informativos, anuncios y/o programas y espacios de infoentretenimiento y que están relacionados con las elecciones de los meses de abril y noviembre de 2019.

Para ello perseguimos los siguientes objetivos secundarios:

- Analizar el nivel de participación e interacción de los millennials en redes sociales con los principales partidos políticos.

- Detectar el tipo de contenidos políticos consumidos por parte de los jóvenes en el medio televisivo.

- Estudiar el nivel de influencia de este tipo de contenidos políticos respecto a los comportamientos y decisiones en las urnas.

- Identificar los tipos de dispositivos y plataformas más usadas por este tipo de audiencia.

- Realizar una comparativa entre los resultados obtenidos en las primeras elecciones del mes de abril, respecto a las segundas del mes de noviembre de 2019 con el fin de determinar si ha habido cambios significativos en el comportamiento de la muestra.

Esta investigación parte de la hipótesis de que la efectividad de los mensajes políticos en las audiencias millennials está relacionada con el nivel de participación e interacción que tengan con los contenidos políticos y según sea su consumo durante las campañas electorales. La elección de las fuentes de información y el medio donde han consumido los contenidos son elementos que influyen en la decisión del voto en las urnas para este tipo de audiencias.

Para ello, se utiliza una metodología cuantitativa a partir del diseño, aplicación y análisis de resultados de una encuesta a jóvenes universitarios de España. La encuesta fue aplicada a través de internet mediante la plataforma Google Forms y para la explotación de datos se utilizó el software SPSS (Statistical Package for Social Sciences). El cuestionario es una técnica cuantitativa consistente en investigar una muestra de sujetos representativos de una población (Hernández-Sanpieri; Fernández-Collado; Baptista-Lucio, 2014). Las ventajas de este método de recolección de datos son que permite obtener información de casi cualquier colectivo, además de que facilita la estandarización de los datos, es decir, se pueden tratar informáticamente y analizarlos estadísticamente. A su vez, al tratarse de un cuestionario online, permite interactuar con el entrevistado, lo que se traduce en menos preguntas incompletas (Díaz-De-Rada, 2012). 
Dado el objetivo general de este análisis, el cuestionario se centró en conocer los hábitos de consumo de contenidos políticos de los jóvenes universitarios que son usuarios en plataformas y dispositivos, en función de diversas variables relacionadas con:

- nivel de participación e interacción con los contenidos de carácter político;

- tipo de consumo en relación con la programación televisiva dedicada a las elecciones generales en las principales cadenas en abierto;

- uso y consumo de contenidos según plataformas y dispositivos móviles;

- eficacia de los contenidos e influencia de los mensajes a la hora de decidir el voto.

Esta aproximación metodológica es la más adecuada para esta investigación, dada la necesidad de describir comportamientos que puedan ser extrapolables a la población objetivo y porque proporciona la posibilidad de comparar resultados entre las elecciones celebradas en los meses de abril y noviembre, un hecho puntual y significativo que aconteció en España durante 2019. El fin era observar si la repetición de elecciones había provocado (o no) cambios sustanciales en el perfil de la muestra.

El cuestionario consta de 29 preguntas cerradas y de respuesta múltiple distribuidas en cinco bloques temáticos. El beneficio de este tipo de cuestionario radica en que las preguntas cerradas necesitan menos esfuerzo para el encuestado y, por lo tanto, el tiempo para completarlo es menor, además de que permite codificar y analizar los datos fácilmente. Este tipo de cuestionario es acorde con el perfil de la muestra, cuyo nivel de concentración en el momento de realizar una única actividad es relativamente baja, de ahí que se haya diseñado para facilitar y enfocar su atención en la participación de este estudio.

Respecto al diseño del contenido, el cuestionario tiene un bloque inicial de cuatro preguntas para conocer el perfil del encuestado y poder clasificarlo en función de su género, edad, comunidad autónoma de residencia y si ha participado o no en las elecciones políticas. En la segunda ronda de encuestas esta pregunta se sustituyó por una de filtro cuyo requisito residía en haber participado en la primera encuesta sobre las elecciones del mes de abril para poder realizar la segunda encuesta sobre las del mes de noviembre. El siguiente bloque de preguntas ahonda sobre hábitos de consumo respecto a contenidos políticos en dispositivos multipantalla y plataformas digitales y está formado por siete preguntas. El tercer bloque se centra en cuestiones más próximas a la programación televisiva dedicada a las elecciones con un total de cinco preguntas, mientras que el bloque cuatro está compuesto por siete preguntas enfocadas al tipo de consumo y el nivel de participación e interacción de contenidos en redes sociales. Finalmente, el último bloque tiene seis preguntas que versan sobre el nivel de eficacia del consumo de contenidos políticos respecto a la decisión última del voto. A partir de los datos recabados de la encuesta se realiza un análisis descriptivo sobre el consumo televisivo y el nivel de interacción y de participación social en redes sociales, comparando los dos períodos electorales que de manera excepcional hubo en España en 2019.

\section{Población y muestra}

La elección de la población de estudio se justifica porque los jóvenes universitarios cuentan con la mayoría de edad, lo que supone que disfrutan del ejercicio pleno de sus derechos políticos, como el del voto. El contacto con los estudiantes universitarios para su participación en la encuesta se realizó a través del personal docente e investigador de las universidades españolas, públicas y privadas, que participaron y colaboraron en esta investigación. El nivel de estudio al que pertenecían la totalidad de los estudiantes encuestados es el de Grado. Se utilizó un muestreo no probabilístico de conveniencia (Losada; López-Feal, 2003), desarrollándose el trabajo de campo en dos fases: la primera durante el mes de mayo y la segunda en el mes de diciembre y ambas en tres comunidades autónomas: Comunidad Valenciana, Comunidad de Madrid y Andalucía.

Esta elección se realizó con el fin de obtener la máxima representatividad, aunque debemos señalar las limitaciones geográficas de esta investigación, ya que nos ha resultado complicada la extracción de una amplia muestra que represente a todo el territorio nacional. Además, el período temporal marcado por la repetición de elecciones en el mes de noviembre de 2019 condicionó ambos períodos de recogida de datos. No obstante, se ha realizado una comparativa de los resultados obtenidos en las dos fases (mayo y diciembre de 2019) coincidiendo ambas con la finalización de los dos procesos electorales. De esta manera, consideramos que el nivel de respuesta alcanzado ha permitido abordar, entender y comparar la complejidad de la cuestión que estudiamos.

La muestra está compuesta por individuos con edades comprendidas entre 18 y 25 años, siendo un $44 \%$ hombres y un $56 \%$ mujeres. Se obtuvieron 796 respuestas en la primera ronda (elecciones del mes de abril) distribuidas por comunidades autónomas y de la siguiente manera: 56\% Comunidad Valenciana, 32\% Andalucía y 12\% Comunidad de Madrid. En la segunda ronda, (elecciones del mes de noviembre) se obtuvieron 781 encuestas, de las cuales el $52 \%$ fueron de la Comunidad Valenciana; el 35\% de Andalucía y el 13\% de la Comunidad de Madrid.

En esta segunda fase se volvió a enviar el cuestionario online con las mismas preguntas que en la primera fase, pero incluyendo una primera pregunta filtro en la que el usuario debía haber participado en el primer estudio para poder contestar el segundo respecto a las elecciones del mes de noviembre. De esta manera manteníamos el anonimato, pero trabajábamos con la misma muestra representativa permitiendo así comparar comportamientos entre unas elecciones y otras. 
El 97\% manifestó la participación en las primeras elecciones y el $95 \%$ en las segundas. Además, cabe señalar que el $85 \%$ y el $92 \%$ respectivamente afirmó haber consultado el programa electoral de los partidos para decidir su voto, por lo que se manifiesta una alta participación electoral y un elevado interés en la selección y el consumo de contenidos de carácter político entre los jóvenes universitarios españoles en las tres comunidades autónomas.

\section{Principales resultados}

A continuación se presentan los principales resultados obtenidos según cada uno de los items estudiados en la muestra.

\subsection{Hábitos de consumo de contenidos políticos en dispositivos multipantalla y plataformas digitales}

En ambos períodos electorales se detecta un alto índice de interés por los contenidos políticos, siendo incluso un poco más elevado en las segundas elecciones (53\% respecto a un $44 \%$ ). Sólo un $10 \%$ en las primeras, y un $8 \%$ en las segundas, afirmaron no estar interesados en este tipo de contenidos. Además, el $98 \%$ del total de la muestra (y en ambos períodos) considera importante ejercer el derecho al voto.

En relación con el tipo de dispositivo utilizado para consumir contenidos políticos y de cara a las elecciones, los encuestados optaron por el uso de:

- teléfono móvil ( $86 \%$ en la primera ronda y $88 \%$ en la segunda);

- televisión (66\% y 75\%);

- ordenador (52\% y $62 \%)$;

- prensa (20\% y $32 \%)$;

- tableta (8\% y $9 \%)$.

No se detectan variaciones importantes entre las primeras y las segundas elecciones respecto al uso de dispositivo. Sin embargo, cuando se les pregunta por el medio utilizado para informarse sobre las propuestas políticas de cada partido, sí que se detectan variaciones significativas respecto a ambos períodos electorales tal y como se refleja en el gráfico 1.

En cuanto al momento del consumo de contenidos políticos, la mayoría de los jóvenes se conecta y accede a la información en horario de noche, entre las 21 y las 00 horas ( $71 \%$ y $77 \%$ respectivamente), y la frecuencia de consulta oscila entre dos y una hora diaria (53\% y $54 \%$ ), y menos de treinta minutos al día ( $24 \%$ y $20 \%$ ). Los tipos de contenido que más interesan a los jóvenes universitarios españoles son:

- debates electorales (71\% y $73 \%$ );

- programas electorales (54\% y 56\%);

- informativos (44\% y $50 \%$ );

- reportajes especializados en política electoral (31\% y $32 \%)$.

Sólo el $6 \%$ en primera ronda, y el $4 \%$ en la segunda, manifiesta no estar interesado en ningún contenido específico.

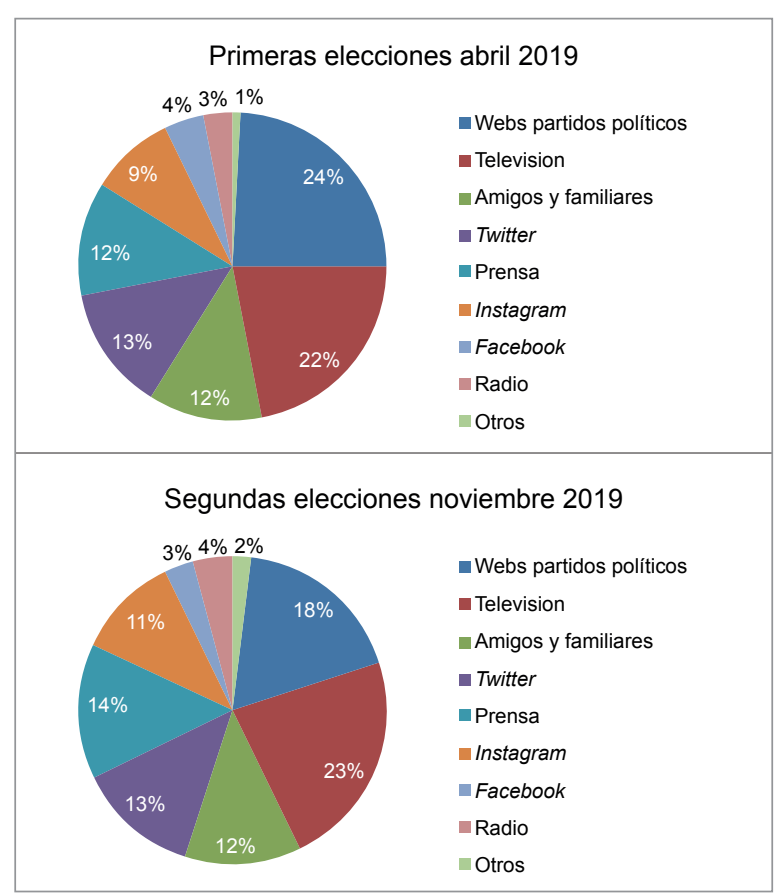

Gráfico 1. Medio utilizado para informarse de las propuestas políticas En ambos períodos electorales la mayoría (92\% y 98\%) prefiere ver los debates en televisión, siendo este el medio favorito. A su vez, más de la mitad de los entrevistados manifestaron que también les gusta seguir los debates en redes sociales de manera simultánea con la televisión ( $52 \%$ y $51 \%)$.

Por tanto, se observa un interés en la búsqueda y en la selección de información por parte de la muestra del estudio.

\subsection{Hábitos de consumo respecto a contenidos políticos dedicados a las elecciones en televisión}

La mayoría de los sujetos, y en ambos períodos electorales, han afirmado que han consumido contenidos con alta frecuencia ( $59 \%$ y $54 \%$ ), el $20 \%$ y $25 \%$ en alguna ocasión y sólo el $14 \%$ y $16 \%$ afirma haberlo hecho de manera indirecta. El $98 \%$ y $95 \%$ respectivamente afirma combinar de manera simultánea y en redes sociales dicho consumo. Por consiguiente, se detecta que a pesar de existir un alto consumo televisivo, estos contenidos se combinan en otras plataformas digitales, especialmente las redes sociales, y de manera simultánea. En cuanto al tipo de contenido favorito consumido en televisión, se observa que en ambos períodos los debates, las noticias relacionadas y las entrevistas a líderes políticos, son los más consumidos por la muestra en el medio televisivo y en ambas elecciones, tal y como se muestra en el gráfico 2.

Los encuestados afirman que han consumido entre uno y dos debates en televisión ( $50 \%$ y $44 \%$ ), un único debate ( $19 \%$ y $26 \%$ ), más de tres debates ( $15 \%$ y $18 \%$ ) y ninguno ( $10 \%$ y $8 \%$ ). Por tanto, se observa un ligero mayor consumo de debates 
electorales en las segundas elecciones respecto a las primeras. La cadena de televisión donde más han visionado los debates en ambos períodos electorales fue La sexta $(52 \%$ y $57 \%$ ), seguida de TVE1 (48\% y 52\%), Antena 3 (46\% y 51\%), Telecinco (17\% y $22 \%)$, Cuatro (14\% y $12 \%)$ y, por último, las autonómicas ( $12 \%$ y $10 \%)$ y TVE2 (2\% y $1 \%)$.

\subsection{Nivel de participación e interacción de conteni- dos políticos en redes sociales}

La mayoría de los encuestados no sigue ni a un partido político (67\% y $64 \%$ ) ni a ningún líder político (63\% y $62 \%)$. No se observan variaciones entre las primeras y las segundas elecciones. Por el contrario, el $56 \%$ sí que ha seguido debates políticos en redes, siendo aún más activo este seguimiento en las segundas elecciones, con un $62 \%$ del total de la muestra. Se detecta un mayor interés en las segundas que en las primeras, al menos en cuanto al seguimiento de los contenidos políticos.

La mayoría de los encuestados ha comentado en redes sociales algún debate político, especialmente en las primeras elecciones (76\%) respecto a las segundas (65\%), pero la mayoría no han compartido esos debates, ni en las primeras (81\%) ni en las segundas (77\%), tal y como se refleja en los gráficos 3 y 4.

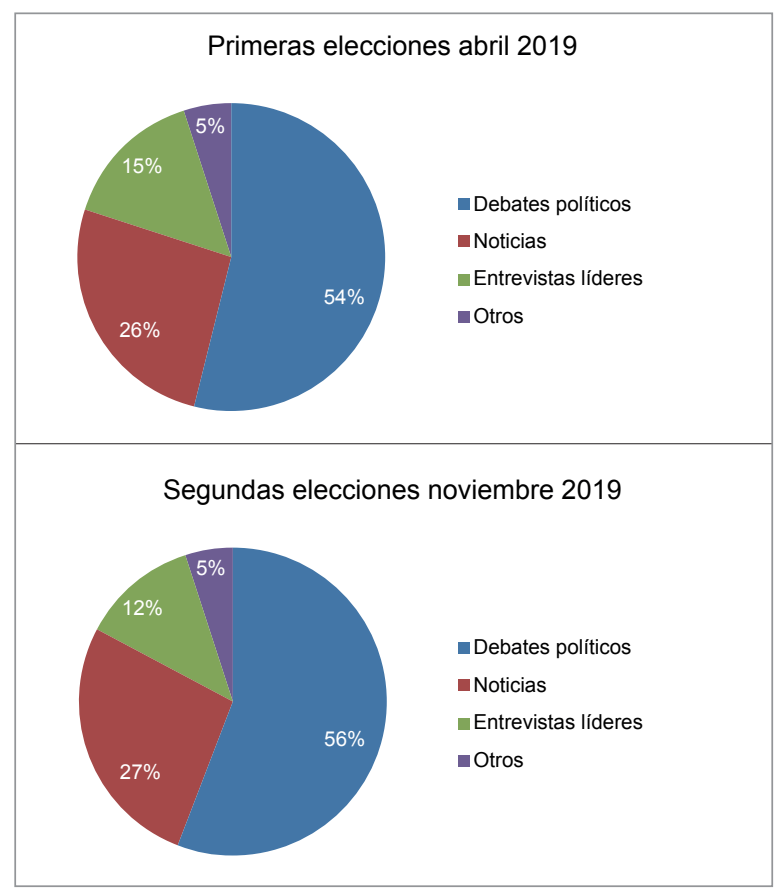

Gráfico 2. Tipo de contenido consumido y relacionado con las elecciones en televisión

\subsection{Nivel de influencia y eficacia de contenidos políticos respecto al comportamiento en las urnas}

En relación con el nivel de influencia de los contenidos políticos a la hora de decidir el voto y, por tanto, el nivel de eficacia de los mismos, se detecta una diferencia notable entre las primeras y las segundas elecciones, tal y como se refleja en el gráfico 5.

Por el contrario, es relevante destacar que tanto en las primeras (54\%) como en las segundas elecciones (58\%), la mayoría de los encuestados considera que los contenidos que han compartido y/o publicado los líderes políticos en sus respectivas redes sociales no les ha influenciado en absoluto a la hora de decidir su voto. En cambio, les han influido más aquellos contenidos de carácter político que han compartido en redes amigos y familiares en ambos períodos electorales, tal como se puede observar en el gráfico 6.

La mayoría de los encuestados manifiesta una necesidad de cambios políticos en España y en ambos períodos electorales ( $95 \%$ y $98 \%$ respectivamente). Además, la mayoría considera que la política afecta en gran medida a sus vidas personales

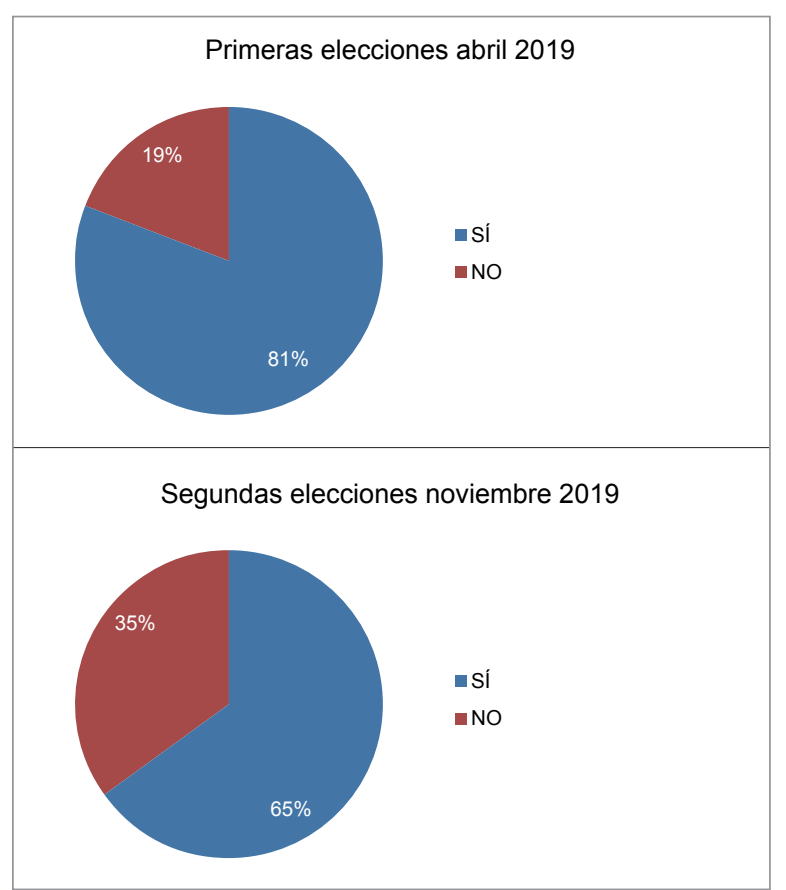

Gráfico 3. ¿Has comentado algún debate político en redes?

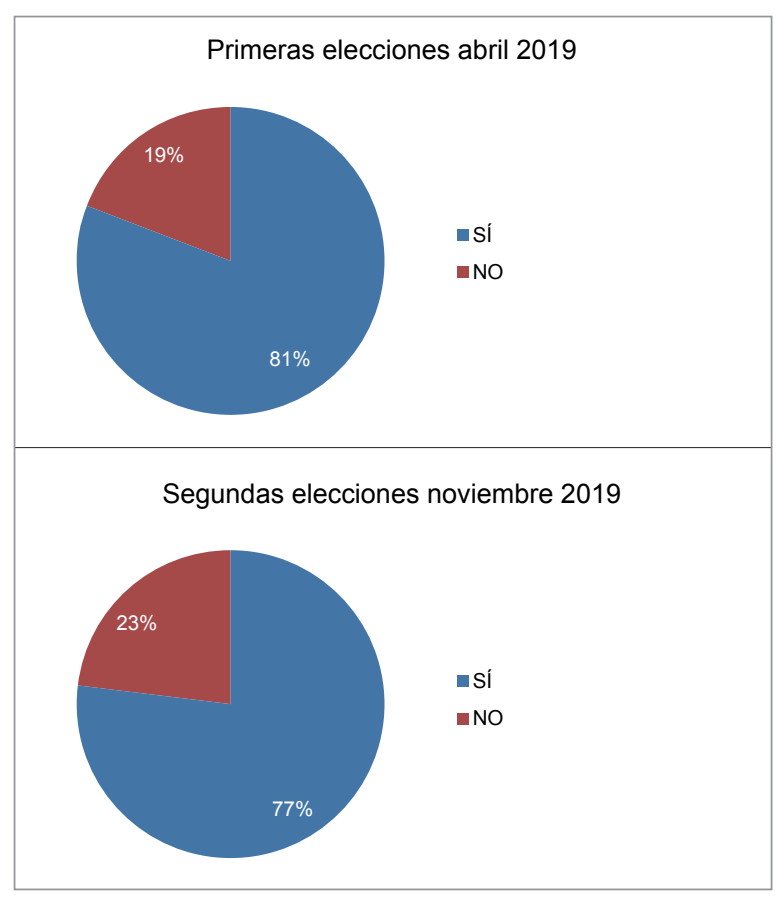

Gráfico 4. ¿Has compartido algún debate político en redes? 


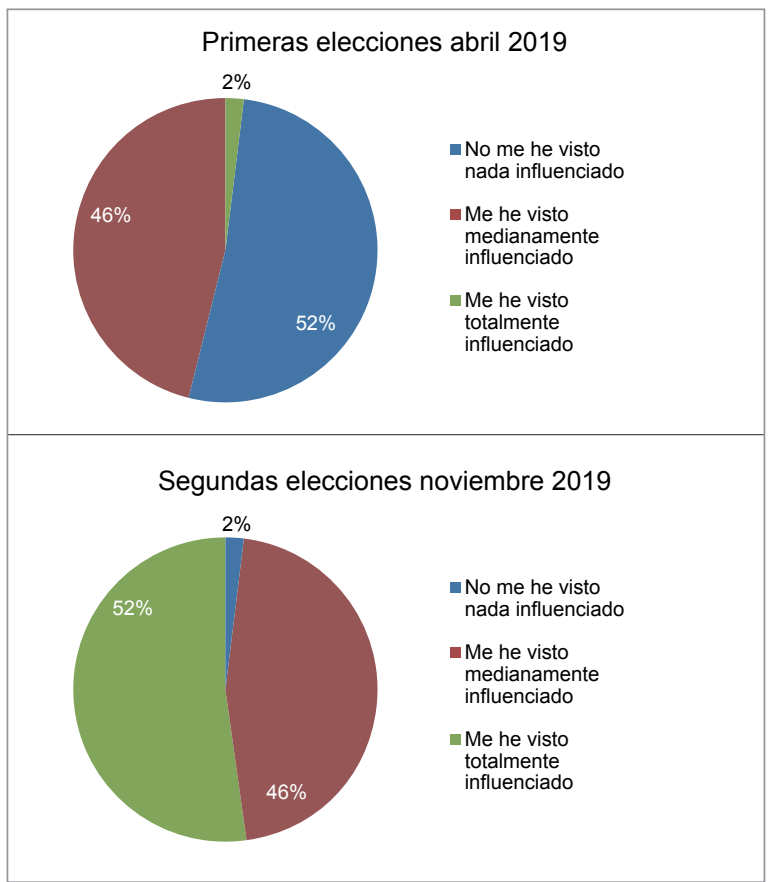

Gráfico 5. Nivel de influencia y eficacia de los contenidos políticos en televisión respecto a la decisión del voto

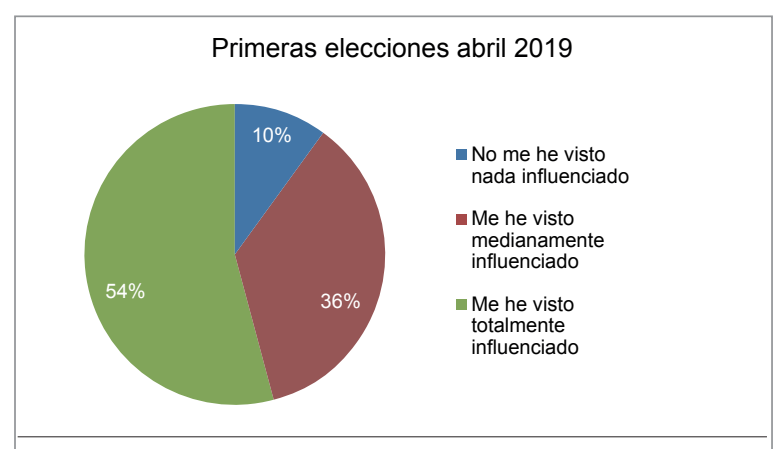

Segundas elecciones noviembre 2019

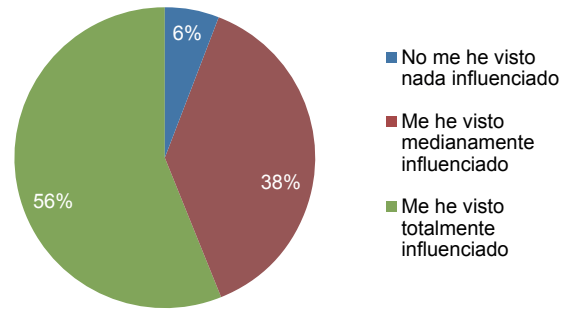

Gráfico 6. ¿En qué medida consideras que te han afectado los contenidos compartidos en redes por tus familiares y/o amigos en tu decisión de voto?

( $64 \%$ en las primeras y $59 \%$ en las segundas), el $18 \%$ y el $21 \%$ levemente, y únicamente el $16 \%$ y el $18 \%$ considera que le afecta más de lo necesario. Por tanto, se observa una alta concienciación sobre la importancia que tiene la política en la actualidad y en cómo esta afecta a la vida de las personas. Por el contrario, la mayoría de los encuestados no confía en el actual sistema político español ( $43 \%$ y $45 \%$ ), el $42 \%$ y el $43 \%$ confía en ocasiones y, sólo el $14 \%$ y el $11 \%$ confía plenamente.

Por último, estos datos contrastan cuando se les pregunta por su nivel de confianza en los dirigentes políticos, existiendo grandes diferencias. En las primeras elecciones de abril la mayoría afirma que sí tienen confianza en los líderes políticos; por el contrario, en las segundas elecciones este dato baja y aumenta notablemente la desconfianza en dichos dirigentes (gráfico 7).

\section{Discusión y conclusiones}

El objetivo principal e hipótesis de partida de nuestra investigación era evidenciar y analizar el nivel de participación social e interacción de las audiencias millennials respecto a los contenidos políticos en redes sociales y en televisión, y comprobar si dicho comportamiento había tenido o no un efecto en sus decisiones dentro de las urnas. Ambos aspectos se han alcanzado: el primero, al mostrar cómo han participado y cómo han interactuado con los contenidos de índole política en ambas elecciones de abril y noviembre de 2019. Se ha detectado una cierta selectividad a la hora de elegir el consumo, las fuentes de información y se han estudiado sus principales comportamientos ante este tipo de contenidos y en diferentes pantallas. También se han recopilado cuáles son sus preferencias de consumo y la manera en que les ha influido a la hora de decidir su voto.

El segundo aspecto, al evidenciar que la efectividad de los mensajes políticos en las audiencias millennials sí que está relacionada con el nivel de participación e interacción que tienen con los contenidos políticos y según sea su consumo durante las campañas electorales, siendo este diferente si se ha producido en las primeras o en las segundas elecciones. Además, hemos podido verificar que la elección de las fuentes de información y el medio donde han consumido los contenidos son elementos que sí que influyen en la decisión del voto en las urnas para este tipo de audiencias. Por tanto, los partidos políticos deben mejorar sus contenidos para alcanzar una mayor efectividad y una mejora de la fidelización ante este perfil de audiencia.

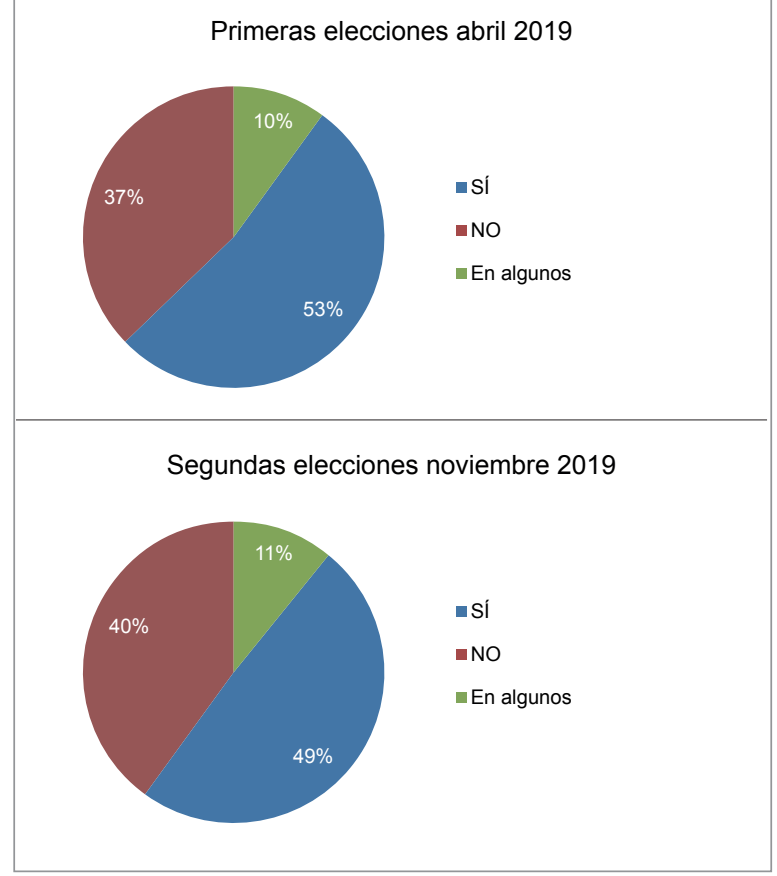

Gráfico 7. ¿Confías en los dirigentes políticos españoles? 
Según los datos obtenidos, los dispositivos que más han utilizado las audiencias millennials en las dos campañas electorales de España en 2019 han sido el teléfono móvil

\section{Las audiencias millennials reivindican un cambio político e inminente en España}

y la televisión, con una alta frecuencia de consumo en horario nocturno y realizándolo de manera simultánea. Es decir, el consumo incluye ambas pantallas al mismo tiempo y mientras en el medio televisivo se centra en contenidos políticos, en las redes sociales y a través de su teléfono móvil, se consumen contenidos de diferentes temáticas. A pesar de ello, los debates políticos en televisión son el contenido más interesante para este perfil de audiencia. Les siguen las noticias y las entrevistas a líderes políticos, cuyo seguimiento se realiza en redes, comentando sobre dichos contenidos. No obstante, no comparten dicho material en sus perfiles. Por tanto, se observa que no manifiestan en público sus preferencias políticas. El consumo aumentó en las segundas elecciones y la cadena de televisión preferida fue La sexta, seguida de TVE1 y Antena 3 en ambos períodos electorales.

El medio favorito para informarse sobre temas políticos son las webs oficiales de los partidos políticos en las primeras elecciones. Por el contrario, en las segundas optan por la televisión como medio más inmediato para acceder a la información. La red social Twitter, y los amigos y familiares, son los otros dos medios favoritos para acceder a la información.

Es interesante destacar que, a pesar de ser una audiencia muy activa en redes sociales, la mayoría de los encuestados afirma no seguir a ningún líder político ni a ningún partido político. De nuevo se detecta cierta cautela a la hora de manifestar en público sus preferencias políticas, así como la no existencia de una fidelización latente a la hora de decidir su voto. De hecho, existe una mayor influencia en el mismo sobre los contenidos políticos en las segundas elecciones, pero no en aquellos donde la fuente proviene de líderes y partidos políticos. Para este tipo de audiencia, la influencia es mayor en las segundas elecciones y cuando la fuente proviene de familiares y amigos respecto a fuentes oficiales emitidas por los medios de comunicación. La influencia es mayor en un entorno relacionado con el familiar y/o de amistades en las redes sociales. En cambio, los contenidos consumidos en televisión, y cuya fuente principal son los propios partidos políticos, tienen una mayor influencia en este perfil de audiencia.

Las audiencias millennials reivindican un cambio político e inminente en España; están más concienciados con la política ya que consideran que afecta a sus vidas y a su futuro. Por el contrario, no confían en el sistema político ni en los líderes actuales de una manera absoluta, siendo más pronunciada dicha desconfianza al repetir las elecciones. Por consiguiente, se observa que dada la coyuntura política de España respecto a la repetición de las elecciones, los contenidos políticos han generado un mayor impacto y, por tanto, una mayor influencia a la hora de decidir el voto final, especialmente en el medio televisivo y en las segundas elecciones. En cambio, en redes sociales los contenidos compartidos por familiares y amigos han influido mucho más que los publicados por los líderes políticos en el medio online. Aun así, las audiencias españolas no tienen una alta implicación en acciones de ciberactivismo, sino que su participación política en redes sociales tiene más un carácter pasivo centrado en el consumo de contenidos (lectura de noticias, visionado de debates, etc.) que comentan pero no comparten con otros usuarios. No obstante, se observa una participación más activa y mucho más selectiva a la hora de localizar los medios para informarse de los contenidos políticos, combinando el medio televisivo y las redes sociales de manera simultánea.

Finalmente, como sinopsis de todo el trabajo, podemos afirmar que esta investigación nos ha mostrado que el sistema político español requiere nuevos planteamientos y nuevas estrategias al confeccionar los contenidos para que sean eficaces en las decisiones de las urnas para este tipo de público. Hemos de tener en cuenta

\section{Se observa una alta concienciación so- bre la importancia que tiene la política en la actualidad y en cómo afecta a la vida de las personas} que la producción y distribución de información política involucra a más actores, medios y plataformas, y las audiencias pueden intervenir de manera más activa e, incluso, influir en la dinámica informativa (Casero-Ripollés, 2018). Por consiguiente, los líderes políticos deben mejorar sus comunicaciones ante este tipo de audiencia para ganar su confianza y su interés, puesto que son audiencias que tienen un ciclo de duración largo al tratarse de jóvenes españoles.

La clave reside en analizar y comprender el comportamiento de los consumidores para adecuar bien la oferta a las necesidades y a los nuevos hábitos de consumo televisivos que la sociedad ha ido adquiriendo, especialmente, en audiencias más jóvenes y en relación con contenidos políticos. Por tanto, se requieren nuevos planteamientos y contenidos que sean mucho más próximos a sus intereses y que provoquen el impacto necesario para que la interacción y la participación de esta audiencia que está tan acostumbrada, sea más explícito en los contenidos políticos.

\section{Referencias}

Álvarez-Monzoncillo, José-María; López-Villanueva, Javier (2017). “Entretenidos, dispares, participativos, empoderados, vigilados y furtivos”. En: Álvarez-Monzoncillo, José-María; De-Haro, Guillermo (coords.). Millennials: La generación emprendedora. Barcelona: Ariel; Fundación Telefónica, pp. 194-222. ISBN: 978840817606

https://www.fundaciontelefonica.com/cultura-digital/publicaciones/588 
Barton, Christine; Koslow, Lara; Beauchamp, Christine (2014). "How millennials are changing the face of marketing forever". BCG perspectives, January 15.

https://cutt.ly/hglzJhi

Beauchamp, Michelle B.; Barnes, Donald C. (2015). "Delighting baby boomers and millennials: Factors that matter most". Journal of marketing theory and practice, v. 23, n. 3, pp. 338-350.

https://doi.org/10.1080/10696679.2015.1032472

Benavides-Delgado, Juan; Villagra-García, Nuria; Alameda-García, David; Fernández-Blanco, Elena (2010). "Los anunciantes españoles y el nuevo contexto de comunicación: una aproximación cualitativa". Revista latina de comunicación social, n. 65, pp. 159-175.

http://www.revistalatinacs.org/10/art/890_UCM/12_Benavides_et_al.html

Bermejo-Berros, Jesús (2008). "El receptor publicitario del siglo XXI en el marco de la interactividad: entre el crosumer y el prosumer". En: Pacheco-Rueda, Marta (coord.). La publicidad en el contexto digital. Sevilla: Comunicación Social. ISBN: 9788496082717

Bustamante, Enrique (2006). La televisión económica. Barcelona, Gedisa. ISBN: 9788474327458

Cabrera-González, María-Ángeles (2010). "La interactividad de las audiencias en entornos de convergencia digital". Icono 14, v. 8, n. 1, pp. 164-177.

https://doi.org/10.7195/ri14.v8i1.287

Campos-Freire, Francisco (2010). El cambio mediático. Zamora: Comunicación Social. ISBN: 9788492860258

Campos-Freire, Francisco (2015). "Adaptación de los medios tradicionales a la innovación de los metamedios". El profesional de la información, v. 24, n. 4, pp. 441-450.

https://doi.org/10.3145/epi.2015.jul.11

Casero-Ripollés, Andreu (2018). "Research on political information and social media: Key points and challenges for the future". El profesional de la información, v. 27, n. 5, pp. 964-974.

https://doi.org/10.3145/epi.2018.sep.01

Castelló-Martínez, Araceli; Del-Pino-Romero, Cristina; Tur-Viñes, Victoria (2016). “Estrategias de contenido con famosos en marcas dirigidas a público adolescente". Icono14, v. 14, n. 1, pp. 123-154.

https://doi.org/10.7195/ri14.v14i1.883

Castells, Manuel (2007). La transición en la sociedad red. Barcelona: Ariel. ISBN: 9788434442719

Castromil, Antón R.; Rodríguez-Díaz, Raquel; Garrigós, Paula (2020). “La agenda política en las elecciones de abril de 2019 en España: programas electorales, visibilidad en Twitter y debates electorales". El profesional de la información, v. 29, n. 2, e290217.

https://doi.org/10.3145/epi.2020.mar.17

Cebrián-Herreros, Mariano (2009). “Comunicación interactiva en los cibermedios”. Comunicar, v. 17, n. 33, pp. 15-24. https://doi.org/10.3916/c33-2009-02-001

Chen, Xinran; Sin, Sei-Ching-Joanna; Theng, Yin-Leng; Lee, Chei-Sian (2015). "Why students share misinformation on social media: Motivation, gender, and study-level differences". The journal of academic librarianship, v. 41, n. 5, pp. 583-592. https://doi.org/10.1016/j.acalib.2015.07.003

Crovi, Delia (2004). Sociedad de la información y el conocimiento. Entre lo falaz y lo posible. Buenos Aires: UNAM; La Crujía Ediciones. ISBN: 9789871004713

Cunningham, Stuart; Craig, David; Silver, Jon (2016). "YouTube, multichannel networks and the accelerated evolution of the new screen ecology". Convergence, v. 22, n. 4, pp. 376-391.

https://doi.org/10.1177/1354856516641620

Dalton, Russell J. (2004). Democratic challenges, democratic choices. The erosion of political support in advanced industrial democracies. Oxford: Oxford University Press. ISBN: 9780199297900

De-Salas-Nestares, María-Isabel (2010). “La publicidad en redes sociales. De lo intrusivo a lo consentido”. Icono 14, v. 8, n. 1. https://doi.org/10.7195/ri14.v8i1.281

DeVaney, Sharon (2015). "Understanding the millennial generation". Journal of financial service professionals, v. $69, \mathrm{n}$. 6, pp. 11-14.

Dias, Patrícia; Teixeira-Botelho, Inês (2016). "Multi-screening: Prácticas emergentes, motivaciones y expectativas". Redes.com, n. 13, pp. 273-292.

https://dialnet.unirioja.es/servlet/articulo?codigo $=5769025$ 
Díaz-De-Rada, Vidal (2012). "Ventajas e inconvenientes de la encuesta por internet". Papers: revista de sociologia, v. 97, n. 1, pp. 193-223.

https://doi.org/10.5565/rev/papers/v97n1.71

Diego-González, Patricia; Guerrero-Pérez, Enrique; Etayo-Pérez, Cristina (2014). "Televisión conectada en España: Contenidos, pantallas y hábitos de visionado". Revista mediterránea de comunicación, v. 5, n. 1, pp. 179-199.

https://doi.org/10.14198/MEDCOM2014.5.1.10

Espinoza, Chip; Ukleja, Mick; Rusch, Craig (2010). Managing the millennials: Discover the core competencies for managing today's workforce. USA: John Wiley and Sons editions.

Ferguson, Douglas A.; Greer, Clark F. (2016). "Reaching a moving target: How local TV stations are using digital tools to connect with generation C". International journal on media management, v. 18, n. 3-4, pp. 141-161. https://doi.org/10.1080/14241277.2016.1245191

Ferreras-Rodríguez, Eva-María (2014). "Los nuevos prosumidores: Audiencias de la televisión social. Análisis de Operación Palace en Twitter". Revista mediterránea de comunicación, v. 5, n. 1, pp. 175-192. https://doi.org/10.14198/MEDCOM2014.5.2.11

Francés-Domenec, Miquel; Llorca-Abad, Germán; Peris-Blanes, Àlvar (2015). La televisión conectada en el entorno transmedia. Pamplona: Eunsa Ediciones. ISBN: 9788431330385

Gallego, Francisco (2013). "Social TV analytics: nuevas métricas para una nueva forma de ver televisión". Index.comunicación, v. 3, n. 1, pp. 13-39.

http://journals.sfu.ca/indexcomunicacion/index.php/indexcomunicacion/article/view/49

Gil, Víctor; Romero, Felipe (2008). Crossumer, claves para entender al consumidor español de nueva generación. Madrid: Ediciones Gestión 2000. ISBN: 9788498750010

González-Neira, Ana; Berrocal-Gonzalo, Salomé; Zamora-Martínez, Patricia (2020). "Fórmulas de emisión y consumo de los debates televisivos en España en las elecciones legislativas de 2019". El profesional de la información, v. 29, n. 2, e290221. https://doi.org/10.3145/epi.2020.mar.21

González-Neira, Ana; Quintas-Froufe, Natalia (2015). "Revisión del concepto de televisión social y sus audiencias". En: Quintas-Froufe, Natalia; González-Neira, Ana (coord.). La participación de la audiencia en la televisión: de la audiencia activa a la social. Madrid: AIMC, pp. 13-26. ISBN: 9788460842422

González-Oñate, Cristina (2008). Nuevas estrategias de televisión: El desafío digital. Madrid: Ciencias Sociales. ISBN: 978 8487510489

Grossman, Lawrence K. (1995). The electronic republic: Reshaping democracy in the information age. New York: Viking. ISBN: 9780140249217

Guerrero-Pérez, Enrique (2018). "La fuga de los millennials de la televisión lineal”. Revista latina de comunicación social, n. 73, pp. 1231-1246.

https://doi.org/10.4185/RLCS-2018-1304

Guerrero-Pérez, Enrique; González-Oñate, Cristina; Kimber-Camussetti, David (2018). “Millennials' TV: An approach to their wiewing habits". Anàlisi: Quaderns de comunicació i cultura, n. 59, pp. 121-138.

https://doi.org/10.5565/rev/analisi.3151

Hernández-Sanpieri, Roberto; Fernández-Collado, Carlos; Baptista-Lucio, Pilar (2014). Metodología de la investigación. México DF: McGraw-Hill Interamericana de México. ISBN: 9781456223960

http://observatorio.epacartagena.gov.co/wp-content/uploads/2017/08/metodologia-de-la-investigacion-sextaedicion.compressed.pdf

Hershatter, Andrea; Epstein, Molly (2010). “Millennials and the world of work: An organization and management perspective". Journal of business and psychology, v. 25, n. 2, pp. 211-223.

https://doi.org/10.1007/s10869-010-9160-y

Jenkins, Henry; Ito, Mizuko; Boyd, Danah (2015). Participatory culture in a networked era: A conversation on youth, learning, commerce, and politics. Cambridge: Polity Press. ISBN: 9781509503230

Kantar TNS (2014). Connected life.

https://www.kantar.com/es

León, Bienvenido; García-Avilés, José-Alberto (2012). “Los retos de la implantación de la televisión interactiva a la luz de su propia historia". Zer, v. 7, n. 13.

https://www.ehu.eus/ojs/index.php/Zer/article/view/6024/5706 
Li, Charlene; Bernoff, Josh (2009). El mundo Grounswell: cómo aprovechar los movimientos sociales espontáneos de la Red. Barcelona: Empresa Activa. ISBN: 9788492452194

Losada, José-Luis; López-Feal, Rafael (2003). Métodos de investigación en Ciencias Humanas y Sociales. Madrid: Thomson. ISBN: 9788497321907

Marta-Lazo, Carmen; Gabelas-Barroso, José-Antonio (2013). "Hábitos de consumo televisivo de ficción entre los universitarios que estudian comunicación". Revista de comunicación de la SEECI, año XVII, n. 31, pp. 14-33.

https://doi.org/10.15198/seeci.2013.31

Masip, Pere; Guallar, Javier; Suau, Jaume; Ruiz-Caballero, Carlos; Peralta, Miquel (2015). “News and social networks: audience behavior". El profesional de la información, v. 24, n. 4, pp. 363-370.

https://doi.org/10.3145/epi.2015.jul.02

Masip, Pere; Ruiz-Caballero, Carlos; Suau, Jaume (2019). “Active audiences and social discussion on the digital public sphere. Review article". El profesional de la información, v. 28, n. 2, e280204.

https://doi.org/10.3145/epi.2019.mar.04

Oliver, Xavier; Alloza, Ángel (2009). Atrapados por el consumo. Madrid: LID. ISBN: 9788483561324

Perilla-Lozano, Leonor; Zapata-Cadavid, Bárbara (2009). "Redes sociales, participación e interacción social”. Trabajo social, n. 11, pp. 147-158.

https://dialnet.unirioja.es/servlet/articulo?codigo $=4085233$

Prensario (2018). Storytelling for millennials, Gen Z... and more. MIP White Paper.

Prensky, Marc (2001). "Digital natives, digital immigrants". On the horizon, v. 9, n. 5, pp. 1-6.

https://marcprensky.com/writing/Prensky\%20-\%20Digital\%20Natives,\%20Digital\%20lmmigrants\%20-\%20Part1.pdf

Quintas-Froufe, Natalia; González-Neira, Ana (2014). “Audiencias activas: participación de la audiencia social en la televisión". Comunicar, v. 22, n. 43, pp. 83-90.

https://doi.org/10.3916/C43-2014-08

Quintas-Froufe, Natalia; González-Neira, Ana (2016). “Consumo televisivo y su medición en España: camino hacia las audiencias híbridas". El profesional de la información, v. 25, n. 3, pp. 376-383.

https://doi.org/10.3145/epi.2016.may.07

Rodríguez-Vázquez, Ana-Isabel; Silva-Rodríguez, Alba; Direito-Rebollal, Sabela; García-Orosa, Berta (2020). “Convenciones y disrupciones en la cobertura de los eventos políticos en la TV lineal y social. Análisis de la noche de las elecciones del 26M de 2019". El profesional de la información, v. 29, n. 2, e290205.

https://doi.org/10.3145/epi.2020.mar.05

Ruiz-Cartagena, José-Javier (2017). “Millennials y redes sociales: estrategias para una comunicación de marca efectiva”. Miguel Hernández communication journal, n. 8, pp. 347-367.

https://dialnet.unirioja.es/servlet/articulo?codigo $=6120205$

Saavedra-Llamas, Marta; Papí-Gálvez, Natalia; Perlado-Lamo-de-Espinosa, Marta (2020). "Televisión y redes sociales: las audiencias sociales en la estrategia publicitaria". El profesional de la información, v. 29, n. 2, e290206.

https://doi.org/10.3145/epi.2020.mar.06

Scolari, Carlos A.; Fernández-de-Azcárate, Sara; Garín, Manuel; Guerrero, Mar; Jiménez, Manel; Martos, Aitor; Obradors, Matilde; Oliva, Mercè; Pérez, Óliver; Pujadas, Eva (2012). “Narrativas transmediáticas, convergencia audiovisual y nuevas estrategias de comunicación". Quaderns del CAC, v. 15, n. 38, pp. 79-89.

https://www.cac.cat/sites/default/files/2019-01/Q38_scolari_et_al_ES.pdf

Tur-Viñes, Victoria (2020). “Television program ratings and informed audiences". El profesional de la información, v. 29, n. 2, e290212.

https://doi.org/10.3145/epi.2020.mar.12

Vaca, Ricardo (2015). "Hacia un nuevo consumo audiovisual: TV e Internet”. En: Francés-Domenec, Miquel; Llorca-Abad, Germán; Peris-Blanes, Àlvar. La televisión conectada en el entorno transmedia. Pamplona: Eunsa Ediciones. ISBN: 978 8431330385

Valiente, Carlos (2016). "Las claves de la nueva publicidad para los millennials". IPMarkt, n. 825, pp. 32-35.

Waddell, T. Franklin; Bailey, Amanda (2017). "Inspired by the crowd: The effect of online comments on elevation and universal orientation". Communication monographs journal, v. 84, n. 4, pp. 534-550.

https://doi.org/10.1080/03637751.2017.1369137

Waddell, T. Franklin; Sundar, S. Shyam (2017). "\#thisshowsucks! The overpowering influence of negative social media comments on television viewers". Journal of broadcasting \& electronic media, v. 61, n. 2, pp. 393-409.

https://doi.org/10.1080/08838151.2017.1309414 